\title{
Factores predictores de aparición de fibrilación auricular en pacientes con miocardiopatía dilatada
}

\author{
Predicting factors of atrial fibrillation in patients with dilated cardiomyopathy \\ Víctor Castro ${ }^{*}$, Jorge Toquero ${ }^{1}$, Luisa Pérez², Jon Orruño ${ }^{3}$, Javier Alzueta ${ }^{4}$, Xabier Viñolas ${ }^{5}$, \\ Luis Álvarez ${ }^{6}$, Ignasi Anguera ${ }^{7}$ e Ignacio Fernández ${ }^{1}$
}

${ }^{1}$ Servicio de Cardiología, Unidad de Electrofisiología, Hospital Universitario Puerta de Hierro Majadahonda, Madrid; ${ }^{2}$ Servicio de Cardiología, Unidad de Electrofisiología, Hospital Juan Canalejo, La Coruña; ${ }^{3}$ Servicio de Cardiología, Unidad de Electrofisiología, Hospital Universitario Araba, Vitoria; ${ }^{4}$ Servicio de Cardiología, Unidad de Arritmias, Hospital Virgen de la Victoria de Málaga, Málaga; ${ }^{5}$ Servicio de Cardiología, Unidad de Electrofisiología, Hospital de la Santa Creu i Sant Pau, Barcelona; ${ }^{6}$ Servicio de Cardiología, Unidad de Electrofisiología, Hospital Nuestra Señora de la Candelaria, Santa Cruz de Tenerife; ${ }^{7}$ Servicio de Cardiología, Unidad de Electrofisiología, Hospital de Bellvitge, L'Hospitalet de Llobregat, Barcelona. España

\section{Resumen}

Introducción: El uso de terapia anticoagulante en pacientes con miocardiopatía dilatada es controvertido. El riesgo hemorrágico hace que habitualmente no se use en pacientes en ritmo sinusal. Objetivo: Analizar los factores predictores de fibrilación auricular (FA) en pacientes con miocardiopatía dilatada y fracción de eyección del ventrículo izquierdo (FEVI) < 40. Método: Se estudiaron los pacientes incluidos en el registro multicéntrico UMBRELLA a quienes se había implantado un desfibrilador (DAl) bicameral o tricameral y que presentaban miocardiopatía dilatada isquémica o no isquémica y FEVI < 40\%. Se definió FA como cualquier episodio > 30 segundos de duración y una frecuencia auricular > 175 latidos por minuto. Resultados: Se incluyeron 684 enfermos. La mediana de edad fue de 70 años (rango intercuartílico [RIQ]: 62-77). El 79.1\% eran varones. La FEVI fue $<30 \%$ en el $76.3 \%$. El $87.3 \%$ presentaban insuficiencia cardiaca (ICC) clínica. Se implantó un DAI resincronizador en el 59.5\%. El 51.2\% tenían bloqueo de rama izquierda del haz de His y el 7.1\% de rama derecha (BRDHH). Se documentó $F A$ en el 49\% de los enfermos con una mediana de seguimiento de 29.93 meses (RIQ: 14.78-45.63). Las variables que se relacionaron con la aparición de FA fueron la presencia de ICC (hazard ratio [HR]: 2; intervalo de confianza del 95\% [IC 95\%]: 1.31-3.04; $p=0.001$ ), el BRDHH (HR: 1.48; IC 95\%: 1-2-18; $p=0.045)$, el ictus previo (HR: 2.11; IC 95\%: 1.4-3.19; $p<0.001$ ) y la edad > 75 años (HR: 1.21; IC 95\%: 1.05-1.40; $p=0.008$ ). Conclusiones: La edad > 75 años, el BRDHH, la ICC y el ictus previo predicen la aparición de FA en la población con miocardiopatía dilatada y FEVI < 40\%.

Palabras clave: Fibrilación auricular. Miocardiopatía dilatada. Anticoagulación. DAl.

\section{Abstract}

Introduction: Anticoagulant treatment in patients with dilated cardiomyopathy and sinus rhythm is controversial due to haemorrhage risk. Objective: To analyze the factors predicting atrial fibrillation $(A F)$ in patients with dilated cardiomyopathy and ejection fraction (LVEF) < 40\%. Method: All patients included in UMBRELLA multicentre registry without AF, who had a dual

\section{Correspondencia:}

*Víctor Castro

E-mail: vcastrou14@yahoo.com
Disponible en internet: 07-01-2022

Rev Colomb Cardiol. 2021;28(6):548-555

www.rccardiologia.com 0120-5633 / @ 2021 Sociedad Colombiana de Cardiología y Cirugía Cardiovascular. Publicado por Permanyer. Este es un artículo open access bajo la licencia CC BY-NC-ND (http://creativecommons.org/licenses/by-nc-nd/4.0/). 
or three-chamber implantable cardiac defibrillator (ICD), dilated cardiomyopathy and LVEF $<40 \%$ were included. AF was defined as any episode $>30$ seconds of duration and atrial frequency $>175 \mathrm{bpm}$. Results: 684 patients were included. Median age was 70 years (IQR 62-77); $79.1 \%$ were male. LVEF was $<30 \%$ in $76.3 \%$ of cases; $87.3 \%$ presented clinical heart failure (CHF). A CRT-D was implanted in 59.5\%; $51.2 \%$ of patients presented Left Bundle Branch Block (LBBB) and 7.1\% presented Right Bundle Branch Block (RBBB). AF was documented in $49 \%$ of patients, with a median follow-up of 29.93 months (IQR: 14.78-45.63). The presence of CHF (HR: 2; 95\% Cl: 1.31-3.04; $p=0.001$ ), RBBB (HR: 1.48; 95\% Cl: 1-2-18; $p=0.045$ ), previous stroke (HR: 2.11; 95\% Cl: 1.4-3.19; $p<0.001$ ) and age > 75 years (HR: 1.21; 95\% Cl: 1.05-1.40; $p=0.008)$ were associated with diagnosis of AF. Conclusions: Age $>75$ years, RBBB, CHF and previous stroke are predictors of AF development in the population with dilated cardiomyopathy and LVEF $<40 \%$.

Key words: Atrial fibrillation. Dilated cardiomyopathy. Anticoagulation. ICD.

\section{Introducción}

El tratamiento anticoagulante en pacientes con insuficiencia cardiaca (ICC) y fibrilación auricular (FA) se encuentra plenamente estudiado y establecido; sin embargo, existen dudas respecto a su uso en pacientes en ritmo sinusal.

Los pacientes con insuficiencia cardiaca o disfunción ventricular presentan una mayor incidencia de eventos tromboembólicos en comparación con la población general. La incidencia de ictus isquémico fue de 18/1000 pacientes durante el primer año tras el diagnóstico de insuficiencia cardiaca, con un incremento de hasta $47 / 1000$ durante un seguimiento de 5 años ${ }^{1}$. El riesgo de ictus y tromboembolia se encuentra incrementado en la fase inicial (<30 días) tras el diagnóstico de insuficiencia cardiaca. En un estudio poblacional, el riesgo se incrementó en 17.4 veces durante el primer mes tras el diagnóstico de insuficiencia cardiaca ${ }^{2}$.

En el estudio SCD-HeFT ${ }^{3}$, donde se incluyeron pacientes con insuficiencia cardiaca en clase funcional (CF) II o III y sin FA, la incidencia de tromboembolia (la mayoría ictus) fue del $4 \%$ a 4 años de seguimiento. En el análisis multivariante, la hipertensión $(p=0.021)$ y la fracción de eyección del ventrículo izquierdo (FEVI) (hazard ratio [HR]: 0.82; intervalo de confianza del 95\% [IC 95\%]: 0.69-0.97 por cada incremento en 5\%) fueron las variables independientes asociadas a riesgo de tromboembolia.

La insuficiencia cardiaca y la disfunción ventricular se asocian al riesgo de aparición de FA. El incremento del riesgo de ictus en la población con insuficiencia cardiaca en ritmo sinusal podría deberse a la presencia de episodios paroxísticos de FA que cursan de forma asintomática ${ }^{4}$.

En el estudio SOLVD ${ }^{5}$, el uso de warfarina se asoció de forma independiente a una reducción en la mortalidad total (HR: 0.76; IC 95\%: 0.65-0.89; $p=0.0006$ ), la cual no se veía influenciada por la presencia de FA, la edad, la FEVI, la etiología ni el grado funcional.

La anticoagulación en pacientes con disfunción ventricular y ritmo sinusal está generalmente desaconsejada, debido a que a pesar de haber demostrado una reducción en la tasa de ictus isquémico no se asocia a una reducción en la mortalidad total y además incrementa el riesgo de hemorragia mayor.

Conocer las variables que se relacionan con el riesgo de aparición de FA en la población con disfunción ventricular sería de interés, ya que en ellos podría estar justificado el uso de tratamiento anticoagulante profiláctico, especialmente fármacos de acción directa, o al menos la búsqueda exhaustiva de FA mediante métodos de monitorización adecuados.

La presencia de dispositivos intracardiacos con electrodo auricular constituye una herramienta altamente sensible y específica para el diagnóstico de $\mathrm{FA}^{6}$. El objetivo del estudio es la búsqueda de las variables predictoras de FA en pacientes con miocardiopatía dilatada, FEVI $<40 \%$ y portadores de desfibrilador bicameral o tricameral.

\section{Método}

El estudio se encuentra desarrollado dentro del marco para la investigación observacional ofrecido por la Scientific Cooperation Platform (SCOOP), fundamentada dentro del registro UMBRELLA, el cual se trata de un registro nacional español voluntario promocionado por la compañía Medtronic, que incluye pacientes portadores de desfibrilador automático implantable (DAl) Medtronic con monitorización remota Carelink, tanto en prevención primaria como secundaria. Se ofrece a cada paciente al que se implanta un DAI Medtronic con capacidad de seguimiento remoto mediante Carelink la posibilidad de inclusión en el registro, previa firma de consentimiento informado. En este registro participan 28 de los 51 centros españoles donde se implantan 
DAI Medtronic con sistema de monitorización remota Carelink. En el momento de redacción del texto se han incluido 3921 pacientes, previa aprobación por los comités éticos de los centros participantes y obtención del consentimiento informado de los pacientes.

La población de estudio incluye todos los pacientes del registro UMBRELLA con el diagnóstico de miocardiopatía dilatada isquémica o no isquémica, con FEVI $<40 \%$ en los que nunca se haya documentado ningún episodio de FA y sean portadores de DAI bicameral o tricameral. Se precisa la presencia de electrodo auricular para el diagnóstico preciso de FA.

Se excluyeron aquellos pacientes con diagnóstico previo electrocardiográfico de algún episodio de FA.

Se define FA como cualquier episodio del alta frecuencia auricular (> 175 I.p.m.) y con una duración de al menos 30 segundos.

Se realizó un análisis de búsqueda de variables clínicas y electrocardiográficas de FA. Se analizaron las siguientes variables: edad, sexo, FEVI, antecedentes de cardiopatía isquémica, infarto de miocardio previo, revascularización miocárdica, insuficiencia cardiaca clínica (signos y síntomas de congestión), CF de la New York Heart Association (NYHA), hipertensión arterial, hipercolesterolemia, tabaquismo, enfermedad arterial periférica, insuficiencia renal crónica, diabetes mellitus, antecedentes de accidente cerebrovascular, tratamiento farmacológico con amiodarona, sotalol o betabloqueantes, tipo de dispositivo implantado (bicameral o tricameral), implante en prevención primaria o secundaria, score de $\mathrm{CHA}_{2} \mathrm{DS}_{2} \mathrm{VASc}$, anchura de QRS, presencia de trastorno de conducción intraventricular, bloqueo de rama derecha del haz de His (BRDHH), bloqueo de rama izquierda del haz de $\mathrm{His}(\mathrm{BRIHH})$ y ritmo de marcapasos.

\section{Análisis estadístico}

Las variables categóricas se expresan como frecuencia y porcentaje, y se comparan mediante la prueba de chi al cuadrado o el test exacto de Fisher. Las variables continuas se representan mediante la media \pm desviación estándar o la mediana y el rango intercuartílico (RIQ), según proceda. Se usó la prueba t de Student o la prueba $U$ de Mann-Whitney para analizar diferencias entre los grupos. Las variables cuantitativas fueron analizadas mediante el test de Kolmogorov-Smirnoff o el test de Shapiro-Wilk para valorar la distribución normal de las mismas.

Se analizó la supervivencia libre de aparición de FA mediante curva de Kaplan-Meier.
Se analizaron las variables predictoras de aparición de FA mediante el test de Fisher o la prueba de chi cuadrado en caso de variables cualitativas, y mediante la prueba t de Student o la prueba U de Mann Whitney en caso de variables cuantitativas. Se realizó un análisis multivariante mediante regresión logística de Cox, donde se incluyeron aquellas variables con una $p<0.1$ en el análisis univariante. Se fueron excluyendo de forma progresiva hacia atrás aquellas variables con mayor valor de $p$ hasta obtener el resultado final del modelo.

Todos los análisis estadísticos se realizaron mediante el programa SPSS 20 Statistics de IBM Corp., Armonk, NY, USA.

\section{Resultados}

Se incluyeron 684 enfermos a los que se implantó un DAl con cable auricular entre septiembre de 2005 y agosto de 2015. Las características basales de la población de estudio se muestran en la tabla 1.

La mediana de edad de la población fue de 70 años (RIQ: 62-77), el $79.1 \%$ eran varones y el $55.3 \%$ presentaron cardiopatía isquémica. La FEVI fue $<30 \%$ en el $76.3 \%$ de los pacientes. El $87.3 \%$ presentaban insuficiencia cardiaca (ICC) clínica. Se implantó un DAI resincronizador en el $59.5 \%$. El $51.2 \%$ de los enfermos tenían BRIHH y el $7.1 \%$ BRDHH. Un 7.4\% tenía ritmo de marcapasos previo al implante.

Durante una mediana de seguimiento de 29.93 meses (RIQ: 14.78-45.63) se documentó FA en el $49 \%$ de los enfermos. La curva de Kaplan-Meier se muestra en la figura 1.

Apareció FA con una mediana de tiempo desde el implante del dispositivo de 9 meses (RIQ: 1-25,25 meses).

Los pacientes en los que se diagnosticó FA presentaban una anchura de QRS mayor (140 ms [RIQ: 90-190] vs. $130 \mathrm{~ms}$ [RIQ: 70-190]; $p=0.044)$, tenían una edad mayor (71 años [RIQ: 54-88] vs. 67 años [RIQ: 51-83]; $p<0.005)$ y tenían unos valores en la escala de $\mathrm{CHA}_{2} \mathrm{DS}_{2}$ VASc superiores (4 [2-6 vs. $3[1-5 ; p<0.005)$

El uso de fármacos antiarrítmicos (betabloqueantes, amiodarona y sotalol) para otras indicaciones diferentes de la profilaxis de FA no incidió en el diagnóstico de FA. Presentaron FA el $47.9 \%$, el $53 \%$ y el $53.8 \%$ de los pacientes, respectivamente, bajo tratamiento con betabloqueantes, sotalol y amiodarona, frente al $52.2 \%$, el $48 \%$ y el $46.9 \%$ de los pacientes que no tomaban dichos fármacos $(p=0.5, p=0.67$ y $p=0.19$, respectivamente). 
Tabla 1. Características basales de la población

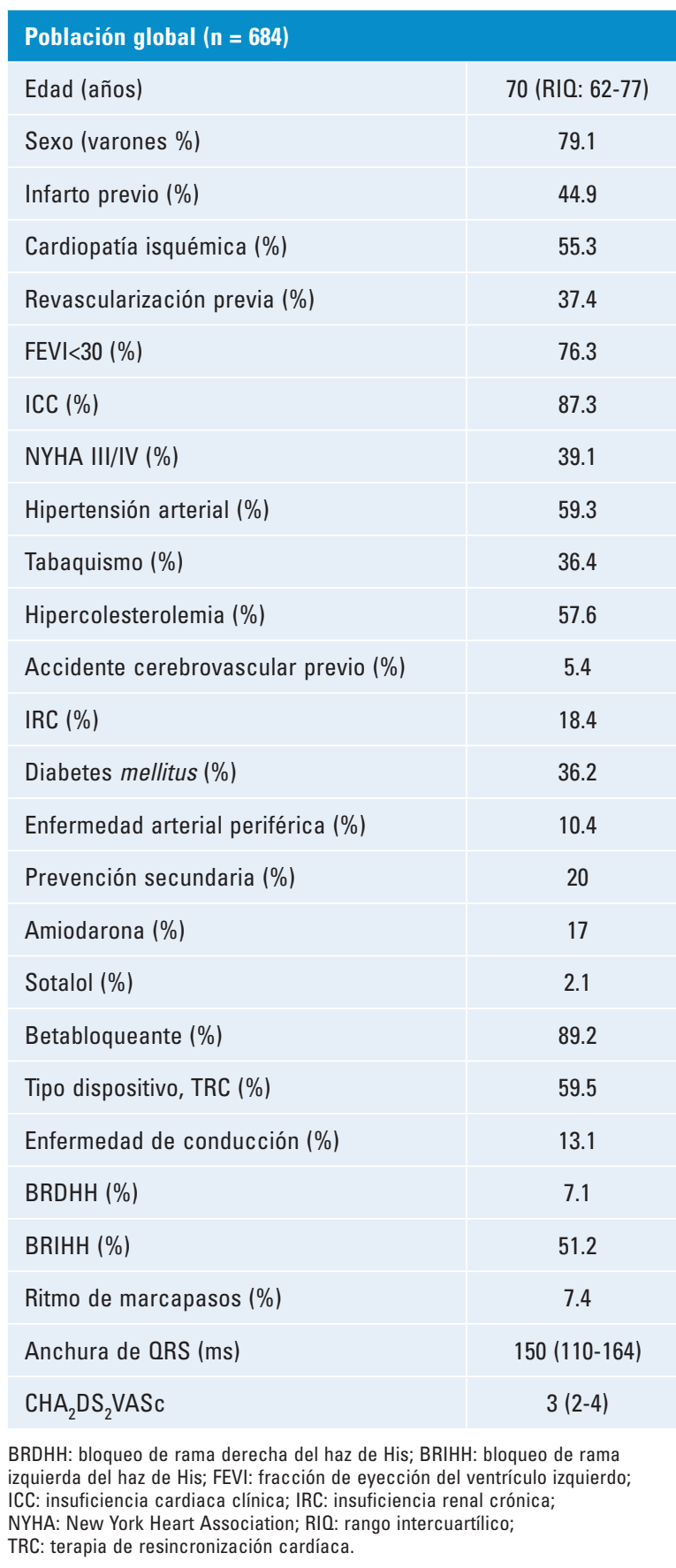

Las características basales de la población según la aparición o no de FA se describen en la tabla 2.

Las variables que se relacionaron con la aparición de FA en el análisis univariante fueron la presencia de ICC clínica (HR: 1.88; IC 95\%: 1.27-2.79 p = 0.002), la presencia de BRDHH (HR: 1.43; IC 95\%: 0.98-2-08 $\mathrm{p}=0.06)$, la insuficiencia renal (HR: 1.31 ; IC $95 \%$ : $1.006-1.71 ; p=0.045)$, la presencia de ictus previo (HR:

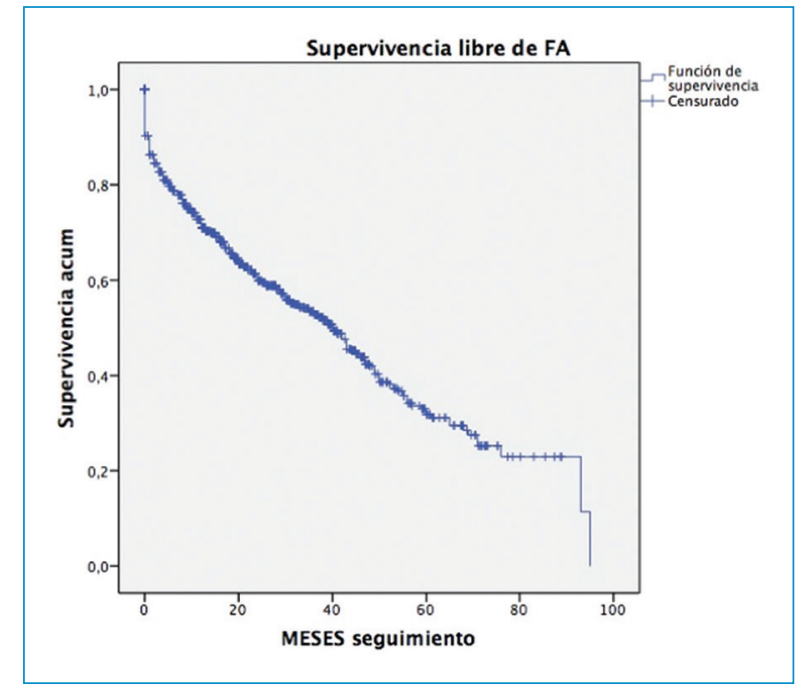

Figura 1. Supervivencia libre de FA.

FA: fibrilación auricular.

2.29; IC 95\%: 1.52-3.46; $p<0.001$ ), la edad > 75 años (HR: 1.25; IC 95\%: 1.1-1-43; $p=0.001$ ), la presencia de escala de $\mathrm{CHA}_{2} \mathrm{DS}_{2}$ VASc > 2 (HR: 1.35; IC 95\%: 1.041.75; $p=0.023$ ), la anchura de QRS (HR: por cada 10 ms 1.04; IC 95\%: 1.01-1.08; $p=0.006$ ) y la edad (HR: por cada 10 años 1.16; IC 95\%: 1.04-1.30; $p=0.006$ ).

El análisis multivariante se muestra en la tabla 3. La presencia de ICC, el BRDHH, el ictus previo y la edad $>75$ años fueron variables predictoras independientes de aparición de FA.

\section{Discusión}

Los pacientes con miocardiopatía dilatada, $\mathrm{FEVI}<40 \%$ y RS, en presencia de ICC clínica, BRD$\mathrm{HH}$, ictus previo o edad $>75$ años tienen más riesgo de aparición de FA. El uso de tratamiento antitrombótico o métodos exhaustivos de búsqueda de FA podrían implementarse en dichos grupos.

Existen estudios aleatorizados que han testado el uso de tratamiento anticoagulante en pacientes con insuficiencia cardiaca y ritmo sinusal. Tres estudios se realizaron antes de 1960, en pacientes hospitalizados y con una tasa elevada de enfermedad valvular reumática $^{7-9}$. En los tres estudios, la terapia anticoagulante se asoció a una reducción en la mortalidad y en la presencia de eventos tromboembólicos. Posteriormente a 1961 se han publicado cuatro estudios aleatorizados $^{10-13}$. En el estudio WASH $^{10}$ se aleatorizó a 279 pacientes a tratamiento con warfarina, ácido acetilsalicílico o no tratamiento. Con un seguimiento medio de 
Tabla 2. Características basales según la aparición o no de fibrilación auricular

\begin{tabular}{|c|c|c|c|}
\hline & $F A(n=335)$ & No FA (n = 349) & $\mathbf{p}$ \\
\hline Edad (años) & $73(58-88)$ & $68(55-81)$ & $<0.005$ \\
\hline Sexo (varones \%) & 75.7 & 78.6 & 0.3 \\
\hline Infarto previo $(\%)$ & 80.9 & 82.6 & 0.63 \\
\hline Revascularización previa (\%) & 67.3 & 71.2 & 0.39 \\
\hline $\mathrm{FEVI}<30(\%)$ & 48.7 & 51.3 & 0.40 \\
\hline ICC $(\%)$ & 81.7 & 71.6 & $<0.005$ \\
\hline NYHA III/IV (\%) & 39.2 & 31.8 & 0.07 \\
\hline Hipertensión arterial (\%) & 55.2 & 54.1 & 0.74 \\
\hline Tabaquismo (\%) & 36.1 & 39.4 & 0.31 \\
\hline Hipercolesterolemia (\%) & 54.7 & 54.6 & 0.99 \\
\hline Accidente cerebrovascular previo (\%) & 7.4 & 3.2 & 0.007 \\
\hline IRC (\%) & 19.5 & 14.3 & 0.04 \\
\hline Diabetes mellitus (\%) & 32 & 31.2 & 0.8 \\
\hline Enfermedad arterial periférica (\%) & 92.1 & 94.3 & 0.35 \\
\hline Prevención secundaria (\%) & 26.8 & 29.9 & 0.3 \\
\hline Amiodarona (\%) & 18.6 & 15.8 & 0.29 \\
\hline Sotalol (\%) & 2.1 & 2 & 0.96 \\
\hline Betabloqueante (\%) & 89.2 & 90.5 & 0.51 \\
\hline Tipo dispositivo, TRC (\%) & 51.8 & 44.3 & 0.07 \\
\hline BRDHH (\%) & 7.9 & 5.2 & 0.1 \\
\hline BRIHH (\%) & 45.8 & 41.1 & 0.15 \\
\hline Ritmo de marcapasos (\%) & 8.6 & 5.1 & 0.06 \\
\hline Anchura de QRS (ms) & 150 (106-194) & $140(86-194)$ & $<0.05$ \\
\hline $\mathrm{CHA}_{2} \mathrm{DS}_{2}$ VASc & $4(2-6)$ & $3(1-5)$ & $<0.05$ \\
\hline
\end{tabular}

BRDHH: bloqueo de rama derecha del haz de His; BRIHH: bloqueo de rama izquierda del haz de His; FA: fibrilación auricular; FEVI: fracción de eyección del ventrículo izquierdo; ICC: insuficiencia cardiaca clínica; IRC: insuficiencia renal crónica; TRC: terapia de resincronización cardíaca.

Tabla 3. Análisis multivariante para aparición de fibrilación auricular

\begin{tabular}{|l|c|c|}
\hline ICC & HR: 2 ; IC 95\%: 1.31-3.04 & $p=0.001$ \\
\hline BRDHH & HR: 1.48 ; IC 95\%: $1.2-18$ & $p=0.045$ \\
\hline Ictus previo & HR: 2.11 ; IC 95\%: $1.4-3.19$ & $p<0.001$ \\
\hline Edad > 75 años & HR: 1.21 ; IC 95\%: 1.05-1.40 & $p=0.008$ \\
\hline
\end{tabular}

BRDHH: bloqueo de rama derecha del haz de His; HR: hazard ratio; IC 95\%: intervalo de confianza del 95\%; ICC: insuficiencia cardiaca clínica.

27 meses, no hubo diferencias significativas en el end point primario de muerte, infarto 0 accidente cerebrovascular. En el estudio $\mathrm{HELAS}^{11}$ se aleatorizaron 197 pacientes de acuerdo con el tipo de cardiopatía. Se comparó el tratamiento con warfarina y ácido acetilsalicílico en los isquémicos y entre warfarina y placebo en los no isquémicos. Nuevamente no hubo diferencias significativas con un seguimiento de 18.521.9 meses. La hemorragia mayor fue más frecuente en el grupo de los pacientes que recibieron warfarina. Por último, en el estudio WATCH ${ }^{12}$ se incluyeron 1587 pacientes, que fueron aleatorizados a tratamiento con warfarina, ácido acetilsalicílico o clopidogrel. No hubo diferencias en el end point combinado de muerte, ictus e infarto de miocardio. Sin embargo, sí se encontró una 
reducción significativa respecto al ictus con el uso de warfarina en comparación con el uso de antiagregantes plaquetarios, pero a costa de un aumento en la tasa de sangrado mayor.

El estudio WARCEF ${ }^{13}$ es el más largo y reciente publicado en pacientes con disfunción ventricular y ritmo sinusal. Se incluyeron 2305 pacientes que fueron seguidos durante un periodo de tiempo de 6 años, sin encontrar diferencias significativas en el end point combinado de ictus isquémico, hemorragia intracraneal o muerte (HR: 0.93; IC 95\%: 0.79-1.10; $p=0.40$ ) entre aquellos aleatorizados a warfarina o a ácido acetilsalicílico. Se observó una reducción significativa con el uso de warfarina en la tasa de ictus isquémico (HR: 0.52; IC 95\%: 0.33-0.82; $p=0.005$ ). Sin embargo, la tasa de hemorragia mayor fue significativamente más alta en el grupo de tratamiento con warfarina, si bien no hubo diferencias significativas en cuanto a la tasa de hemorragia intracraneal.

En nuestro estudio y con una mediana de seguimiento de 29.93 meses (RIQ: 14.78-45.63) se diagnosticó FA en casi el $50 \%$ de los enfermos, lo que implica una incidencia altísima y una probabilidad muy destacable de desarrollo de FA y, por tanto, de necesidad de tratamiento anticoagulante. Además, de acuerdo con la tendencia en la curva de supervivencia, es de esperar que la tasa de FA continúe incrementándose proporcionalmente al aumento del tiempo de monitorización.

La variable principal del estudio fue la aparición de FA de duración $\geq 30$ segundos, lo cual es consistente con las guías de práctica clínica en ablación de $\mathrm{FA}^{14}$, y aunque no deja de ser un valor arbitrario, es un hallazgo fundamental para la valoración de la necesidad de anticoagulación del paciente. En la actualidad, todavía se discute cuál es el valor de duración necesario que implica la necesidad de tratamiento anticoagulante (30 segundos, 5 minutos, 24 horas, etc.).

Los factores independientes predictores de aparición de FA fueron la presencia de insuficiencia cardiaca, tener antecedentes de haber presentado ictus, ser mayor de 75 años y mostrar en el electrocardiograma de superficie un BRDHH.

Es sabido que la prevalencia de FA en pacientes con insuficiencia cardiaca es alta, y que además se incrementa a medida que progresa el grado de insuficiencia cardiaca. Así, en estudios clásicos de fármacos, la prevalencia de FA se incrementó de un $4.2 \%$ en $\mathrm{CF}$ I de la NYHA hasta un $10-15 \%$ en CF II-III, un $26-30 \%$ en CF II-IV y hasta un $49.8 \%$ en CF IV ${ }^{15-20}$.

En dichos estudios, los episodios de FA se obtenían mediante electrogramas de superficie, por lo que la prevalencia está claramente infraestimada. El implante de dispositivos intracardiacos permite la posibilidad de una monitorización constante, precisa y exacta (en caso de presencia de cable auricular), lo que constituye un escenario ideal para el estudio de dicha patología en esta población.

Casi el $40 \%$ de nuestra población de estudio presentaba una CF III-IV de la NYHA. Si bien la presencia de insuficiencia cardiaca clínica sí se mostró como predictora independiente del desarrollo de FA, no fue así la presencia de CF de la NYHA avanzadas. Posiblemente, la mayor capacidad diagnóstica por el mayor tiempo de monitorización mediante dispositivos implantables hace que no existan diferencias en cuanto a incidencia de FA entre CF avanzadas y poco avanzadas de la NYHA; al contrario, como se pone de manifiesto por diferentes estudios mediante monitorización por electrograma de superficie.

El $20 \%$ de los ictus isquémicos son de origen cardioembólico. Cuando su causa es desconocida se denomina ictus criptogénico, lo cual ocurre en el $20-40 \%$ de los casos tras un protocolo extenso de estudio ${ }^{21-25}$. Una de las causas más frecuentes es la $\mathrm{FA}^{26}$, y el riesgo de recurrencia se reduce drásticamente con el uso de la anticoagulación ${ }^{26,27}$.

En pacientes con ictus criptogénico, la monitorización prolongada (durante 36 meses) mediante Holter insertable ${ }^{28}$ ha demostrado una incidencia de aparición de FA de hasta el $30 \%$, muy superior a la obtenida mediante seguimiento convencional. De modo similar, la incidencia de FA fue del $16.1 \%$ en el subgrupo de pacientes que fueron seguidos mediante registrador de eventos por un periodo de 30 días $^{29}$ tras la presencia de un ictus criptogénico.

La FA es muy poco frecuente o casi inexistente en niños y adolescentes, y cuando existe casi siempre se asocia con la presencia de alguna enfermedad cardiaca estructural o canalopatía. La prevalencia de FA aumenta progresivamente con la edad ${ }^{30-32}$. La relación entre edad y FA quedó de manifiesto en el estudio ATRIA $^{33}$. En este estudio poblacional que incluyó 1.9 millones de personas, la prevalencia global fue del $1 \%$, el $70 \%$ de los pacientes con FA tenían al menos 65 años y el $45 \%$ una edad $\geq 75$ años. La prevalencia en menores de 55 años fue del $0.1 \%$, en contraposición con el $9 \%$ en aquellos con edad $\geq 80$ años. De forma similar, en un estudio poblacional europe ${ }^{32}$ la prevalencia global de FA fue del $5.5 \%$, variando entre el $0.7 \%$ en aquellos de $55-59$ años hasta el $17.8 \%$ en aquellos con edad $\geq 85$ años. 
La asociación entre BRDHH y aparición de FA es un hallazgo novedoso no reflejado en la literatura con anterioridad. En un estudio poblacional realizado en Dinamarca ${ }^{34,35}$, la presencia de BRDHH se asoció de manera independiente con el sexo masculino, la edad y un menor índice de masa corporal. Si bien en dicho estudio la presencia de BRDHH se asoció con riesgo de padecer infarto de miocardio y necesidad de marcapasos, dichos pacientes no presentaron más riesgo de insuficiencia cardiaca o FA. Aunque en nuestro estudio se asoció de manera independiente con la aparición de FA, podría existir cierta asociación con la edad que explicara dichos hallazgos.

Por tanto, en aquellos pacientes con miocardiopatía dilatada y $\mathrm{FEVI}<40 \%$, portadores de un dispositivo altamente sensible y específico para el diagnóstico de FA, se llega al diagnóstico en uno de cada dos pacientes, en especial en la población que presenta insuficiencia cardiaca clínica, BRDHH y edad $>75$ años. Es por tanto que en este grupo poblacional se hace necesaria la intervención mediante medios diagnósticos avanzados (registradores de eventos, Holter insertable, etc.) para el diagnóstico precoz y el empleo inmediato de medicación anticoagulante, con una mejora pronóstica evidente. Sería planteable, o al menos discutible, el empleo profiláctico de medicación anticoagulante, especialmente con fármacos de acción directa, con menor riesgo hemorrágico, en aquellos pacientes con características relacionadas de forma independiente con el desarrollo de FA.

\section{Limitaciones}

Se trata de un estudio descriptivo en el que múltiples variables han sido analizadas, pero no se puede descartar la presencia de sesgos o de otras variables que no hayan sido tenidas en consideración. No fue recogido en el estudio el empleo de inhibidores de la enzima convertidora de la angiotensina, antagonistas de los receptores de la angiotensina II o antialdosterónicos, los cuales podrían haber tenido algún efecto en el resultado, si bien es improbable, ya que en el caso del uso de medicación betabloqueante no se hallaron diferencias.

El punto de corte para el diagnóstico de FA se estableció en 30 segundos, lo cual es un valor arbitrario, pero ampliamente recogido en las guías de ablación de FA para definir una recurrencia. Dado que se trata de una población de alto riesgo embólico, estimamos que incluso episodios de corta duración pueden tener valor pronóstico, y por tanto así se definió el evento. La aplicación de otros puntos de corte más elevados podría haber arrojado una incidencia menor de FA y resultados diferentes.

\section{Conclusiones}

La edad > 75 años, el BRDHH, la ICC y el ictus previo predicen la aparición de FA en la población con miocardiopatía dilatada y $\mathrm{FEVI}<40 \%$. En dichos pacientes se hace imprescindible la búsqueda intensiva de FA y podría estar justificada la anticoagulación profiláctica incluso en RS.

\section{Financiamiento}

El estudio no cuenta con ningún financiamiento.

\section{Conflicto de intereses}

Los autores no presentan conflicto de intereses.

\section{Responsabilidades éticas}

Protección de personas y animales. Los autores declaran que para esta investigación no se han realizado experimentos en seres humanos ni en animales.

Confidencialidad de los datos. Los autores declaran que han seguido los protocolos de su centro de trabajo sobre la publicación de datos de pacientes.

Derecho a la privacidad y consentimiento informado. Los autores declaran que en este artículo no aparecen datos de pacientes.

\section{Bibliografía}

1. Witt BJ, Gami AS, Ballman KV, Brown R, Meverden R, Jacobsen SJ, Roger $\mathrm{V}$. The incidence of ischemic stroke in chronic heart failure: a meta-analysis. J Card Fail. 2007;13:489-96.

2. Witt BJ, Brown RD Jr, Jacobsen SJ, Weston SA, Ballman KV, Meverden RA, et al. Ischemic stroke after heart failure: a community based study. Am Heart J. 2006;152:102-9.

3. Freudenberger RS, Hellkamp A, Halperin JL, Poole J, Anderson J, Johnson G, et al.; SCD HeFT Investigators. Risk of thromboembolism in heart failure: an analysis from the Sudden Cardiac Death in Heart Failure Trial (SCD-HeFT). Circulation. 2007;115:2637-41.

4. Healey JS, Connolly SJ, Gold MR, Israel CW, Van Gelder IC, Capucci A, et al.; ASSERT Inverstigators. Subclinical atrial fibrillation and the risk of stroke. N Engl J Med. 2012;366:120-9.

5. Al Khadra AS, Salem DN, Rand WM, Udelson JE, Smith JJ, Konstam MA. Warfarin anticoagulation and survival: a cohort analysis from the studies of left ventricular dysfunction. J Am Coll Cardiol. 1998;31:749-53.

6. Plummer CJ, McComb JM. Detection of atrial fibrillation by permanent pacemakers: observations from the STOP AF trial. Card Electrophysiol Rev. 2003;7:333-40.

7. Anderson GM, Hull E. The effects of dicumarol upon the mortality and incidence of thromboembolism complication in congestive heart failure. Am Heart J. 1950;39:697-702.

8. Harvey WP, Finch CA. Dicoumarol prophylaxis of thromboembolism disease in congestive heart failure. N Engl J Med. 1950;242:208-11.

9. Griffith GC, Strangnell R, Levinson DC, Moore FJ, Ware AG. A study of the beneficial effects of anticoagulant therapy in congestive heart failure. Ann Intern Med. 1952;37:867-87. 
10. Cleland JGF, Findlay I, Jafri S, Sutton G, Falk R, Bulpitt C, et al. The warfarin/ aspirin study in heart failure (WASH): a randomized trial comparing antithrombotic strategies for patients with heart failure. Am Heart J. 2004;148:157-64.

11. Cokkinos DV, Haralabopoulos GC, Kostis JB, Toutouzas PK. Efficacy of antithrombotic therapy in chronic heart failure: the HELAS study. Eur $\mathrm{J}$ Heart Fail. 2006;8:428-32.

12. Massie BM, Collins JF, Ammon SE, Amstrong PW, Cleland JG, Ezekowitz $\mathrm{M}$, et al. Randomized trial of warfarin, aspirin and clopidogrel in patients with chronic heart failure: the warfarin and antiplatelet therapy in chronic heart failure (WATCH) trial. Circulation. 2009;119:1616-24.

13. Homma S, Thompson J, Pullicino P, Levin B, Freudenberger, Teerlink JR et al. Warfarin and aspirin in patients with heart failure and sinus rhythm. N Engl J Med. 2012;266:1859-69.

14. Calkins H, Kuck KH, Cappato R, Brugada J, Camm AJ, Chen SA, et al. 2012 HRS/EHRA/ECAS expert consensus statement on catheter and surgical ablation of atrial fibrillation: recommendations for patient selection, procedural techniques, patient management and follow up, definitions, endpoints, and research trial design: a report of the Heart Rhythm Society (HRS) Task Force on Catheter and Surgical Ablation of Atrial Fibrillation. Heart Rhythm. 2012;9:632-96.

15. The SOLVD Investigators. Effect on enalapril on survival in patients with reduced left ventricular ejection fraction and congestive heart failure. N Engl J Med. 1991;325:293-302.

16. Carson PE, Johnson GR, Dunkman WB, Fletcher RD, Farrell L, Cohn JN. The influence of atrial fibrillation on prognosis in mild to moderate heart failure. The V-HefT Studies. The V-HeFT VA Cooperative studies group. Circulation. 1993;87:102-10.

17. Deeddania PC, Singh BN, Ellenbogen K, Fisher S, Fletcher R, Singh SN. Spontaneous conversion and maintenance in sinus rhythm by amiodarone in patients with heart failure and atrial fibrillation: observations from the Veterans Affairs Congestive Heart Failure Trial of Antiarrhythmic Therapy (CHF-STAT). Circulation. 1998;98:2574-9.

18. Torp-Pedersen C, Moller M, Bloch-Thompsen PE, Kober L, Sandoe E, Egstrup $\mathrm{K}$, et al. Dofetilide in patients with congestive heart failure and left ventricular dysfunction N Engl J Med. 1999;341:857-65.

19. Doval HC, Nul DR, Grancelli HO, Varini SD, Soifer S, Corrado G, et al. Randomized trial of low dose amiodarone in severe congestive heart failure. Grupo de estudio de la sobrevida en la insuficiencia cardiaca en Argentina (GESICA). Lancet. 1994;344:493-8.

20. The Consensus Trial Study Group. Effects of enalapril on mortality in severe congestive heart failure. Results of the Cooperative North Scandinavian Enalapril Survival Study (CONSENSUS). N Engl J Med. 1987;316:1429-35.

21. Grau AJ, Weimar C, Buggle F, Heinrich A, Goertler M, Neumaier S, et al Risk factors, outcome, and treatment in subtypes of ischemic stroke: the German Stroke Data Bank. Stroke. 2001;32:2559-66.
22. Kolominsky-Rabas PL, Weber M, Gefeller O, Neundoerfer B, Heuschmann PU. Epidemiology of ischemic stroke subtypes according to TOAST criteria: incidence, recurrence, and long term survival in ischemic stroke subtypes: a population based study. Stroke. 2001;32:2735-40.

23. Schsulz UG, Rothwell PM. Differences in vascular risk factors between etiological subtypes of ischemic stroke: importance of population based studies. Stroke. 2003;34:2050-9.

24. Amarenco P, Bogousslavsky J, Caplan LR, Donnan GA, Wolf ME, Hennerici MG. The ASCOD phenotyping of ischemic stroke (updated ASCO phenotyping). Cerebrovasc Dis. 2013;36:1-5.

25. Adams HP Jr, Bendixen BH, Kappelle LJ, Biller J, Love BB, Gordon DL, et al. Classification of subtype of acute ischemic stroke: definitions for use in a multicenter clinical trial. Stroke. 1993;24:35-41.

26. Jauch EC, Saver JL, Adams HP Jr, Bruno A, Connors JJ, Demaerschalk BM, et al. Guidelines for the early management of patients with acute ischemic stroke: a guideline for healthcare professionals from the American Heart Association/American Stroke Association. Stroke. 2013;44:870-947.

27. Healey JS, Connolly SJ, Gold MR, Israel CW, Van Gelder IC, Capucci A, et al. Subclinical atrial fibrillation and the risk of stroke. N Engl J Med. 2012;366:120-9

28. Sanna T, Diener HC, Passman RS, Di Lazzaro $V$, Bernstein RA Morillo CA, et al. Cryptogenic stroke and underlying atrial fibrillation. N Engl J Med. 2014;370:2478-86.

29. Gladstone DJ, Spring M, Dorian P, Panzov V, Thorpe KE, Hall J, et al. Atrial fibrillation in patients with cryptogenic stroke. N Engl J Med. 2014;370:2467-77

30. Majeed A, Moser K, Carroll K. Trends in the prevalence and management of atrial fibrillation in general practice in England and Wales, 1994-1998: analysis of data from the general practice research database. Heart. 2001;86:284.

31. Feinberg WM, Blackshear JL, Laupacis A, Kronmal R, Hart RG. Prevalence, age distribution, and gender of patients with atrial fibrillation. Analysis and implications. Arch Intern Med. 1995;155:469.

32. Heeringa J, van der Kuip DA, Hofman A, Kors JA, van Rooij FJ, Lip GY, et al. Prevalence, incidence and lifetime risk of atrial fibrillation: the Rotterdam study. Eur Heart J. 2006;27:949.

33. Go AS, Hylek EM, Phillips KA, Chang Y, Henault LE, Selby JV, et al. Prevalence of diagnosed atrial fibrillation in adults: national implications for rhythm management and stroke prevention: the AnTicoagulation and Risk Factors in Atrial Fibrillation (ATRIA) Study. JAMA. 2001;285:2370.

34. Bussink B, Holst A, Jespersen L, Deckers JW, Jensen GB, Prescott E, et al. Right bundle branch block: prevalence, risk factors, and outcome in the general population: results from the Copenhagen Heart Study. Eur Heart J. 2013;34:138-46.

35. Fernández Lozano I, Brugada J. Right bundle branch block: are we looking in the right direction? Eur Heart J. 2013;34:86-8. 